\title{
Pneumopericardium, pneumothorax, and pulmonary hemorrhage from invasive pulmonary aspergillosis. A case report
}

\author{
Billy C Wang, Richard P Lion* and Harsha K Chandnani \\ Department of Pediatrics, Division of Critical Care, Loma Linda University, CA, USA
}

\begin{abstract}
Pneumopericardium is a rare condition with etiologies including adjacent infection, pathology, or trauma. Invasive pulmonary aspergillosis occurs almost exclusively in immunocompromised patients and may be associated with pneumopericardium. We present a 12-year-old female with acute lymphoblastic leukemia who developed multifocal pulmonary Aspergillus terreus after induction chemotherapy and prolonged granulocytopenia. Despite aggressive antifungal therapy, she developed pneumopericardium, which progressed to pneumothorax and pulmonary hemorrhage, resulting in death. To our knowledge, no reports involving the sequential development of these three findings are reported to date. Unusual to her case was the development of pneumopericardium without fistula or direct evidence of Aspergillus pericarditis.
\end{abstract}

\begin{abstract}
Abbreviations: ARF: Acute respiratory failure; CT: Computed tomography; LamB: Liposomal amphotericin B; IPA: Invasive pulmonary aspergillosis; PH: Pulmonary hemorrhage; PICU: Pediatric intensive care unit; PPC: Pneumopericardium; PPV: Positive pressure ventilation; PTX: Pneumothorax

\section{Background}

Invasive pulmonary aspergillosis (IPA) occurs almost exclusively in immunocompromised patients or those with preexisting pulmonary disease [1]. Rare complications include pneumopericardium (PPC), pneumothorax (PTX) and pulmonary hemorrhage (PH), usually in isolation or paired combination [2-4]. To our knowledge, no case of IPA causing PPC, PTX, and PH together has been reported to date.
\end{abstract}

\section{Case presentation}

A 12-year-old female with pre-B-cell acute lymphoblastic leukemia complicated by pancreatitis, Pseudomonas sepsis, presumed IPA, and induction failure presented to our pediatric intensive care unit (PICU) with acute respiratory failure (ARF) one week after failed induction chemotherapy. Chest computed tomography (CT) prior to PICU arrival revealed multifocal pneumonia (Figure 1) and serum Aspergillus antigen was noted to be positive. Treatment included broad-spectrum antimicrobials including Liposomal Amphotericin B (LAmB). On arrival to the PICU she was initiated on bilevel positive airway pressure and continued treatment with LAmB rather than Voriconazole due to her recent pancreatitis and medication side effect profile. Because of persistent febrile neutropenia and ARF, micafungin was added on PICU day 4. She responded to this treatment with resolution of fever and improvement of ARF by PICU day 7 .

On PICU day 13, our patient developed sudden onset of sharp, substernal chest pain. Chest radiograph and repeat CT of the chest confirmed newly developed PPC in the setting of new cavitary lesions suggestive of aspergillomas (Figure 2). Echocardiogram revealed normal biventricular function and was negative for pericardial effusion or tamponade. Antifungal therapy was transitioned to Voriconazole in light of these clinical findings. Her respiratory status remained stable and serial chest radiographs showed stable PPC.

On PICU day 19, our patient acutely developed hypoxemia and hemoptysis requiring intubation with positive pressure ventilation (PPV). Although her post-intubation radiograph did not reveal PTX, CT chest performed within 2 hours demonstrated a new left sided PTX (Figure 3) requiring thoracostomy tube placement. A percutaneous CT guided biopsy was performed with initial microscopy demonstrating filamentous fungi suspicious for Aspergillus spp. Due to severity of her ARF and need for PPV she had persistent PTX, air-leak, and progressive PPC. On day 24, she developed PH leading to hemorrhagic shock and cardiopulmonary arrest with inability to recover spontaneous circulation.

On post-mortem review, finalized fungal cultures speciated Aspergillus terreus. Autopsy findings revealed diffuse IPA with extensive angioinvasion and $\mathrm{PH}$. The pericardium was found to be intact without fistula to any adjacent cavitation. No fungal elements or growth from the pericardial tissue or fluid was noted post-mortem. The heart, including the valves, myocardium, and endocardium, were normal on gross and histologic examination.

${ }^{\star}$ Correspondence to: Richard Lion, DO, Loma Linda University Children's Hospital, Department of Pediatrics, Division of Critical Care, 11234 Anderson Street, Room 5763, Loma Linda, CA 92354, USA, E-mail: richardpliondo@ gmail.com

Key words: aspergillosis, pneumopericardium, pneumothorax, pulmonary hemorrhage

Received: May 09, 2020; Accepted: May 13, 2020; Published: May 15, 2020 


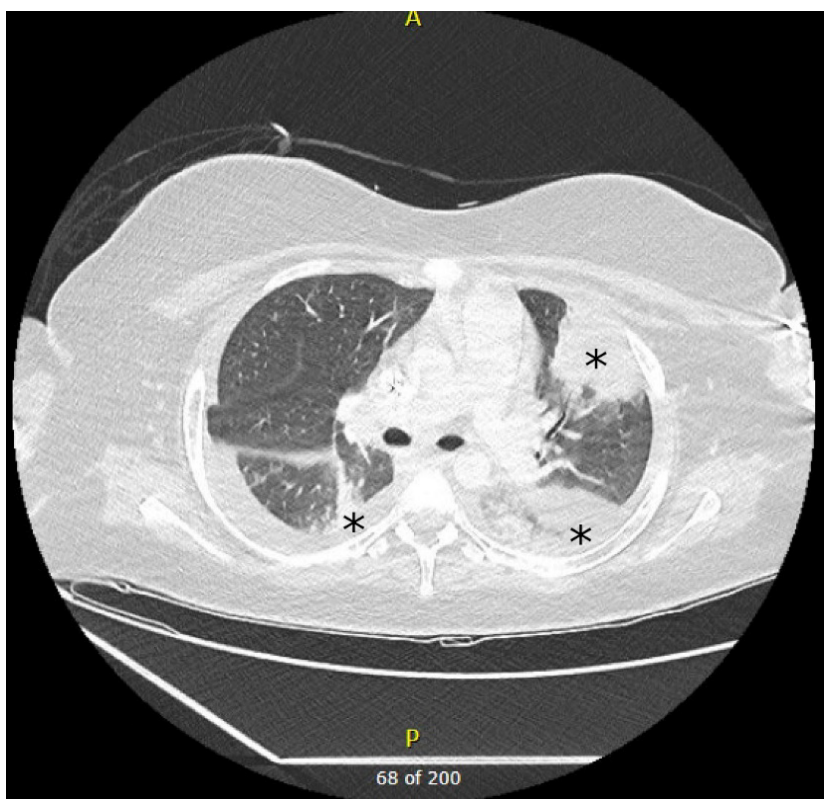

Figure 1. CT findings with infiltrates suggestive of multifocal pneumonia (black asterisks)

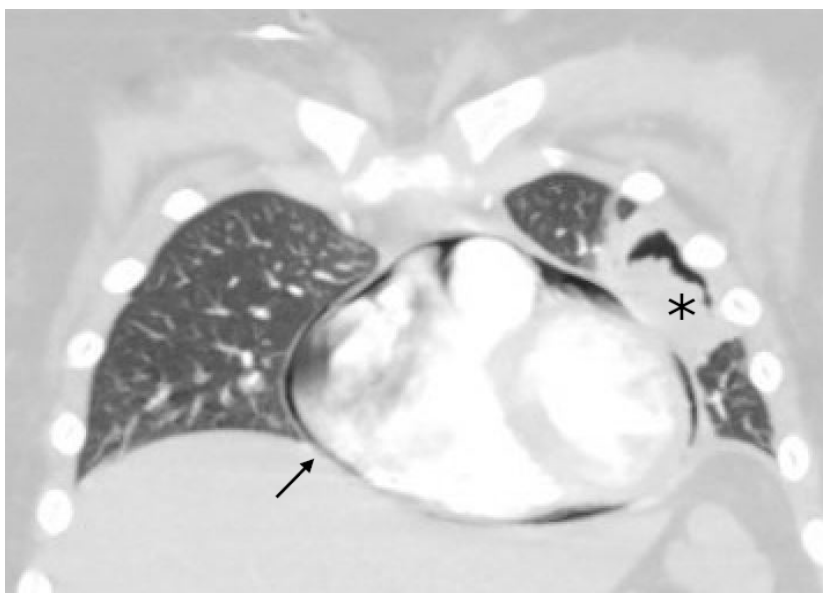

Figure 2. Aspergilloma adjacent to pericardium (black asterisk) with pneumopericardium (black arrow)



Figure 3. Chest CT demonstrating pneumothorax (white asterisk) with adjacent aspergilloma (black asterisk) and expanding pneumopericardium

\section{Discussion}

Our patient died from devastating PH, PTX, and PPC, each known to be complications associated with IPA [2-5]. Given the severe pancreatitis encountered during induction chemotherapy and persistent pancreatic inflammation noted on CT abdomen prior to PICU admission, Voriconazole, the treatment of choice for Aspergillus terreus, was initially avoided. While this organism represents a relatively small percentage of invasive aspergillosis, most isolates are resistant to LAmB [1]. Unfortunately, final speciation resulted after her death, with no post-mortem antifungal sensitivity analysis performed. The resolution of her acute respiratory failure and initial clinical improvement from PICU day 4 to 7 suggested therapeutic efficacy of the chosen antifungal regimen.

Pediatric PPC is an uncommon clinical finding resulting from heterogenous disease processes including trauma, infection, and various iatrogenic causes [6]. While PPC plus PTX and PPC plus $\mathrm{PH}$ has been described to coexist, to the authors' knowledge, this is the first report of PPC, PTX, and PH complicating a single case of IPA [3,5]. Cases of PPC associated with aspergillus can result from communication with localized infection or infection of cardiac tissue; postmortem histology may confirm invasion, fistula formation, or dissemination from hematogenous aspergillosis [2,4,5]. Although we initially suspected pericardial invasion or fistula formation, unusual to this case was lack of these findings or endomyocardial involvement on autopsy. Microscopy did not reveal fungal elements and culture failed to reveal growth within the small volume of pericardial fluid; however, aspergillus antigen detection of this fluid was not performed by our pathologist.

Management of uncomplicated PPC is conservative and requires treatment of the underlying source; prophylactic drainage is usually not recommended. If there is evidence of tamponade, an intervention is required to prevent further hemodynamic decompensation [6]. Prompt recognition of rare complications associated with IPA is essential in the overall management of patients.

We postulate that the origins of the pericardial air were from damaged alveoli and lung parenchyma. Microcommunications may occur between the pleural and pericardial cavity as both membranes originate from the lateral plate mesoderm [7]. Previous studies propose that air escaping from alveolar rupture can dissect along the perivascular sheaths towards the lung hilum and dissect into the potential space at the reflection of the visceral and parietal pericardium near the pulmonary venous ostia. These anatomic features could explain PPC enlargement with invasive positive pressure ventilation, and the potential progression to PTX [8]. Lethal PH was the unfortunate culmination of progressive disease and cardiopulmonary injuries in this immunocompromised patient.

\section{Conclusions}

This case highlights the lethality of IPA in the immunocompromised patient and necessary vigilance in infection screening, prevention, and management. A high index of suspicion is required, given the time to positivity of cultures or markers of invasive fungal infection. Choice of antifungal therapy may be limited due to patient co-morbidities. Patients irresponsive to medical therapy may require increasingly hazardous diagnostic and therapeutic options such as biopsy and surgery, each not without its own risk for morbidity and mortality. Complications such as PPC, PTX, and PH secondary to IPA require prompt recognition and management. 


\section{References}

1. Patterson TF, Thompson GR, Denning DW, Fishman JA, Hadley S, et al. (2016) Practice Guidelines for the Diagnosis and Management of Aspergillosis: 2016 Update by the Infectious Diseases Society of America. Clin Infect Dis 63: e1-e60.

2. Le Moing V, Lortholary O, Timsit JF, Couvelard A, Bouges-Michel C, et al. (1998) Aspergillus Pericarditis with Tamponade: Report of a Successfully Treated Case and Review. Clin Infect Dis 26:451-459.

3. Merino JM, Díaz MA, Ramirez M, Ruano D, Madero L (1995) Complicated Pulmonary Aspergillosis with Pneumothorax and Pneumopericardium in a Child with Acute Lymphoblastic Leukemia. Pediatr Hematol Oncol 12: 195-199.
4. van Ede A, de Pauw BE, Meis JFGM, Koot RAC, Heystraten FMJ (1994) Pneumopericardium complicating invasive pulmonary aspergillosis: Case report and review. Infection 22: 102-105.

5. Muller N, Miller R, Ostrow D, Nelems B, Vickars L (1987) Tension pneumopericardium an unusual manifestation of invasive pulmonary aspergillosis. Am J Roentgenol 148: 678-680.

6. Cummings RG, Wesly RLR, Adams DH, Lowe JE (1984) Pneumopericardium Resulting in Cardiac Tamponade. Ann Thorac Surg 37: 511-518.

7. Schoenwolf GC, Bleyl SB, Brauer PR, Francis-West PH (2015) Development of the respiratory system and body cavities. In: Larsen's Human Embryology. Philadelphia, PA: Elsevier/Churchill Livingstone; 2015: 251-266.

8. Wintermark M, Schnyder P (2001) The Macklin Effect. Chest 120: 543-547.

Copyright: $\subset 2020$ Wang BC. This is an open-access article distributed under the terms of the Creative Commons Attribution License, which permits unrestricted use, distribution, and reproduction in any medium, provided the original author and source are credited. 